\title{
A New Joint Friction Model for Parameter Identification and Sensor-less Hand guiding in Industrial Robots
}

\author{
Guanghui Liu \\ Faculty of Robot Science and Engineering, Northeastern University, Shenyang, China \\ Qiang Li \\ Neuroinformatics Group, Center for Cognitive Interaction Technology (CITEC), Bielefeld University, Bielefeld, \\ Germany \\ Lijin Fang
}

Faculty of Robot Science and Engineering, Northeastern University, Shenyang, China, and

Bing Han and Hualiang Zhang

Key Laboratory of Industrial Control Network and System, Shenyang Institute of Automation, Chinese Academy of Sciences,

Shenyang, China

\begin{abstract}
Purpose - In the present article, a new joint friction model is proposed, which can accurately model the real friction, especially in cases with sudden changes in the motion direction. The identification and sensor-less control algorithm are investigated to verify the validity of this model.

Design/methodology/approach - The proposed friction model is nonlinear and it considers the angular displacement and angular velocity of the joint as a secondary compensation for identification. In the present study, we design a pipeline - including a manually designed excitation trajectory, a weighted least squares algorithm for identifying the dynamic parameters and a hand guiding controller for the arm's direct teaching.

Findings - Compared with the conventional joint friction model, the proposed method can effectively predict friction factors during the dynamic motion of the arm. Then friction parameters are quantitatively obtained and compared with the proposed friction model and the conventional friction model indirectly. It is found that the average Root Mean Square Error of predicted six joints in the proposed method decreases by more than $54 \%$. The arm's force control with the full torque employing the estimated dynamic parameters is qualitatively studied. It is concluded that a light-weight industrial robot can be dragged smoothly by the hand guiding.

Practical implications - In the present study, a systematic pipeline is proposed for identifying and controlling an industrial arm. The whole procedure has been verified in a commercial 6 DOF industrial arm. Based on the conducted experiment, it is found that the proposed approach is more accurate in comparison with conventional methods. A hand-guiding demo also illustrates that the proposed approach can provide the industrial arm with the full torque compensation. This essential functionality is widely required in many industrial arms such as kinaesthetic teaching.

Originality/Value - Firstly, a new friction model is proposed. Based on this model, identifying the dynamic parameter is carried out to obtain a set of model parameters of an industrial arm. Finally, a smooth hand guiding control is demonstrated based on the proposed dynamic model.
\end{abstract}

Keywords-Industrial robot, Parameter identification, Friction model, Weighted least squares, Direct teaching.

\section{Introduction}

As an important subject of the human-robot interaction, robot direct teaching is widely studied. Typical robot direct teaching methods require accurate robot dynamic parameters, such as the gravity compensation or forcefree control (Xiao et al., 2019; Xiao et al., 2018). Furthermore, the robot collision detection is widely used in tasks where robots interact with humans. This is typically implemented based on robot dynamic parameters (Sami et al., 2017). Moreover, accurate dynamic parameters allow robots to execute some tasks that require high precision and high dynamic responses (Wu et al., 2010). However, these applications require an accurate understanding of the robot's dynamic parameters. Unfortunately, manufacturers often do not provide or only partially provide dynamics information of the robot. Therefore, experimental identification or calibration is the only reliable way to obtain these types of information.

For n-DOF series robots in the real environment, the friction directly affects the control accuracy and smoothness of the robot arm. Accordingly, it is of 
significant importance to investigate the impact of the friction on the robot performance. Reviewing the literature indicates that several review papers have been issued in this regard (Olsson et al.,1998; Bona et al., 2005). They summarized the approaches for modelling the friction force in robots and discussed different associating factors e.g. displacement and relative velocity of the contact bodies, properties of the surface materials and the presence of lubrication. Moreover, Nevmerzhitskiy et al. (2019) studied the effect of temperature on the joint's friction. Bittencourt et al. (2010) found that the load is an important factor in the friction model. Waiboer et al. (2005) demonstrated that the relative velocity is the most important factor in the joint friction model, which follows the conventional and simplified friction model. In this model, the friction is considered as the Coulomb friction, which is related to the motion direction and viscous friction, having a linear correlation with the velocity. To this end, a new friction model is proposed and nonlinear factors will be introduced in this regard. These factors are related to the velocity to improve the accuracy of the friction prediction.

Most widely adopted methods for identifying dynamic parameters are the least square estimation (Jin et al., 2015) and maximum likelihood estimation (Swevers et al., 1997). Least square estimation is a more robust method so that it is more adopted. Reviewing the literature shows that diverse modifications have been conducted on this approach. More specifically, Kubus et al. (2008) proposed a non-line recursive total least squares estimation for arm's inertial parameters. Meanwhile, Gautier et al. (2013) proposed a nonlinear least squares optimization approach to estimate model parameters of two DOF SCARA robot prototype. Since the least square approach is robust and it is widely used in industrial applications, this approach is adopted in the present study to estimate parameters of the link's inertial and joint friction model. Because the output torques of joints in the majority of industrial arms are not the same, a weighted least square estimation method will be utilized rather than the classical one.

The main contributions of this article can be summarized as the following:

- A new joint friction model is proposed, which can predict the friction more accurately in comparison with conventional models.

- The weighted least square method is adopted for identifying the robot dynamic model, and a more accurate result will be achieved.

- A hand guiding experiment is carried to evaluate the two aforementioned contributions.

The contents of this article are organized as follows: The proposed friction model and the corresponding dynamic parameters are introduced in section 2 . Then details of the excitation trajectory design are discussed in section 3. Furthermore, a robot hand guiding controller is designed based on the identified dynamic model for the arm in section 4 . The proposed friction model and the identification accuracy compared to the conventional friction model is evaluated in section 5. A hand guiding experiment is also carried out to show the practicability of the whole parameter identification method.

\section{Identifying dynamic parameters}

\subsection{Establishment of the robot dynamic model}

The robot parametric dynamic model can be obtained by either the Newton-Euler method or Lagrangian method in the form below (Craig., 2005):

$$
\tau=M(q) \ddot{q}+C(q, \dot{q}) \dot{q}+G(q)+\tau_{f}
$$

where $M(q) \in R^{n \times n}$ and $\mathrm{n}$ are the symmetric inertia matrix and the number of joints, respectively. Moreover, $C(q, \dot{q}) \in R^{n \times n}$ and $G(q) \in R^{n \times 1}$ denote the Coriolis centrifugal force matrix and the vector of gravitational torques, respectively. Moreover, $q, \dot{q}, \ddot{q}$ are $n \times 1$ vectors that determine the angular displacement, angular velocity and angular acceleration of joints in the joint space. Meanwhile, $\tau \in R^{n \times 1}$ and $\tau_{f} \in R^{n \times 1}$ are the driving torque of joints and the frictional torque in the conventional dynamics model, respectively. The linear structure of friction can be expressed in the form below:

$$
\tau_{f}=f_{c} \operatorname{sgn}(\dot{q})+f_{v}(\dot{q})
$$

where $f_{c}$ and $f_{v}$ are the Coulomb and viscous friction coefficients, respectively. However, studies show that this single linear model has a distinct difference with the real friction phenomena. In order to fit the negative slope phenomenon at low speed so that it can be combined with the Coulomb viscous model, a modified friction model is proposed as follows.

$$
\tau_{f}= \begin{cases}|\dot{q} / \varepsilon| f_{c} \operatorname{sgn}(\dot{q}) & |\dot{q}|<\varepsilon \\ f_{c} \operatorname{sgn}(\dot{q})+f_{v}(\dot{q}) & |\dot{q}| \geq \varepsilon\end{cases}
$$

where $\varepsilon$ is the threshold of joint velocity. The viscous friction model can be expressed as:

$$
f_{v}(\dot{q})=f_{v 3} \dot{q}^{3}+f_{v 2} \dot{q}^{2}+f_{v 1} \dot{q}
$$

where $f_{v 1}, f_{v 2}$ and $f_{v 3}$ are real coefficients. 
In the proposed model, the Coulomb friction performs in both positive and negative directions. When the robot joint moves in different directions, the gravitational force is applied to the joint in the opposite direction of the gravity. Accordingly, two Coulomb friction coefficients $f_{c 1}$ and $f_{c 2}$ are considered in equation (4). On the other hand, it is an enormous challenge to accurately define static friction and the friction at low speed. Compared with equation (2), the threshold in equation (3) is set to make the low-speed movement and the commutation movement of joints more smoothly. Furthermore, the velocity square and velocity cube of joints are considered in the calculations to guarantee the accuracy of the proposed friction model.

\subsection{Linearization of the robot dynamic model}

Swevers et al. (2007) demonstrated that the dynamic model in equation (1) can be rewritten into a linear form by a standard parameter linearization as follows:

$$
\tau=Y_{s}(q, \dot{q}, \ddot{q}) \beta_{s}
$$

where $Y_{s}(q, \dot{q}, \ddot{q}) \in R^{n \times n N_{s}}$ is a regression matrix; $\beta_{s}$ is the $n N_{s} \times 1$ vector of standard parameters; $N_{s}$ is the number of dynamic parameters in each joint. Equation (3) indicates that each joint contains 16 dynamic parameters.

$$
\begin{aligned}
\beta_{s}= & {\left[\beta_{s}^{1}, \beta_{s}^{2}, \cdots, \beta_{s}^{n}\right]^{T} } \\
\beta_{s}^{i}= & {\left[m_{i}, m x_{i}, m y_{i}, m z_{i}, I_{x x}^{i}, I_{x y}^{i}, I_{x z}^{i}, I_{y y}^{i},\right.} \\
& \left.I_{y z}^{i}, I_{z z}^{i}, J_{i}, f_{c 1}^{i}, f_{c 2}^{i}, f_{v 1}^{i}, f_{v 2}^{i}, f_{v 3}^{i}\right]^{T}
\end{aligned}
$$

where $\beta_{s}^{i}$ contains the mass of joint $i$ called $m_{i}$. Moreover, $m x_{i}, m y_{i}$ and $m z_{i}$ are the first-order inertia moments of joint $i$, and $I_{x x}^{i}, I_{x y}^{i}, I_{x z}^{i}, I_{y y}^{i}, I_{y z}^{i}, I_{z z}^{i}$ are the inertial tensors of joint $i$ relative to the origin of the joint space coordinate system $i ; J_{i}$ is the inertia moment of the robot and gear of the motor $i ; f_{c 1}^{i}, f_{c 2}^{i}$ denote the positive and negative direction coefficients of the Coulomb friction, respectively. Finally, $f_{v 1}^{i}, f_{v 2}^{i}, f_{v 3}^{i}$ are viscous friction coefficients.

Combining equations (1) and (5) yields the following expression:

$$
\tau=f(q, \dot{q}, \ddot{q}, \beta)=Y_{s} \beta_{s}
$$

In order to simplify the model, while maintaining the model generality, the $j$ th $\left(1 \leq j \leq N_{s}\right)$ dynamic parameter of the $i$ th $(1 \leq i \leq n)$ link is set to 1 and the rest elements of $\beta_{s}^{i}$ are set to 0 . Applying the NewtonEuler method to compute the $\tau$ vector, the $j$ th column elements of the matrix $Y_{s}$ can be obtained from the following expression:

$$
y_{j}=\left(\tau_{1}, \tau_{2}, \cdots \tau_{n}\right)^{T}
$$

It is worth noting that elements of other columns of the matrix $Y_{s}$ can be obtained in the same way. When the robot operates under a random trajectory, multiple matrices $Y_{s}$ can be obtained. Finally, all of matrices $Y_{s}$ are combined to form a matrix $W \in R^{M n \times n N_{s}}$ ( $M$ is the number of $Y_{s}$ matrices). It is necessary to normalize the matrix prior to the decomposition.

$$
\bar{W}_{M n \times n N_{s}}=\bar{Q} \bar{R}
$$

where $\bar{W} \in R^{M n \times n N_{s}}$ is the normalized matrix of the matrix $W$. When nonzero terms of the main diagonal elements of the matrix $\bar{R}$ are moved forward, the matrix can be divided into two parts.

$$
\bar{R}=\left[\begin{array}{ll}
\bar{R}_{1} & \bar{R}_{2}
\end{array}\right]
$$

The corresponding columns of the matrix $\bar{W}_{M n \times n N_{s}}$ are ordered in the same way as the matrix $\bar{R}$.

$$
\bar{W}_{M n \times n N_{s}}=\left[\begin{array}{ll}
\bar{W}_{1} & \bar{W}_{2}
\end{array}\right]
$$

where the matrix $\bar{W}_{1}$ will be used to generate the excitation trajectory. Similarly, matrix $W^{\prime} \in R^{M n \times n N_{s}}$ can be obtained by running the robot under the excitation trajectory so that it can be decomposed as

$$
W_{M n \times n N_{S}}^{\prime}=\left[\begin{array}{ll}
W_{1}^{\prime} & W_{2}^{\prime}
\end{array}\right]
$$

Similarly, $\beta_{s}$ can be divided into two parts.

$$
\begin{gathered}
\beta_{s}=\left(\begin{array}{ll}
\beta_{s}^{\prime} & \beta_{s}^{\prime \prime}
\end{array}\right)^{T} \\
\tau=\left[\begin{array}{ll}
W_{1}^{\prime} & W_{2}^{\prime}
\end{array}\right]\left[\begin{array}{l}
\beta_{s}^{\prime} \\
\beta_{s}^{\prime \prime}
\end{array}\right]
\end{gathered}
$$


According to equations (10) and (11):

$$
W_{2}^{\prime}=k W_{1}^{\prime}
$$

where $k=R_{2} R_{1}^{-1}$. Subsequently, $\tau$ can be rewritten in the form below:

$$
\begin{aligned}
& \tau=W_{1}^{\prime}\left(\beta_{s}^{\prime}+k \beta_{s}^{\prime \prime}\right) \\
& \tau=Y_{b}(q, \dot{q}, \ddot{q}) \beta_{b}
\end{aligned}
$$

where $Y_{b}=W_{1}^{\prime}$ and $\beta_{b}$ is the minimum parameters, which is defined as:

$$
\beta_{b}=\beta_{s}^{\prime}+k \beta_{s}^{\prime \prime}
$$

\subsection{Weighted least squares estimation}

The maximum torques of robot joints highly differ from each other. Taking the ROKAE BX7 robot as an example, the first joint's maximum torque (i.e. the output of reducer) is more than $80 \mathrm{Nm}$, but the sixth joint's maximum torque is less than $8 \mathrm{Nm}$. Accordingly, estimation of the least squares cannot be an unsatisfying solution because each joint is treated equally (Matthias et al., 2015).

Then the error expression $e$ is defined as:

$$
e=Y_{b}(q, \dot{q}, \ddot{q}) \beta_{b}-\tau
$$

The weighted matrix is as follows:

$$
\hat{e}=H e
$$

where $H=\underset{i=1 \cdots n}{\operatorname{diag}}\left\{\frac{1}{\left\|\tau_{M}^{i}\right\|}\right\}$ is the weighted matrix and $\left\|\tau_{M}^{i}\right\|$ is the $l_{2}$-norm of M-dimension output torque vector of joint $i$.

$$
\begin{gathered}
L\left(\beta_{b}\right)=\frac{1}{2} e^{T} H^{T} H e \\
\left\{\frac{\partial L\left(\beta_{b}\right)}{\partial \beta_{b}}\right\}^{T}=Y_{b}^{T} H^{T} H \tau-Y_{b}^{T} H^{T} H Y_{b} \beta_{b}=0
\end{gathered}
$$

The weighted least square equation can be expressed as:

$$
\beta_{b}=\left(Y_{b}^{T} H^{T} H Y_{b}\right)^{-1} Y_{b}^{T} H^{T} H \tau
$$

It should be indicated that the accuracy of the identification result is evaluated through the root mean square error (RMSE) indicator, which is defined as:

$$
R M S E=\sqrt{\frac{\sum_{i=1}^{M}\left(\tau_{c a l}^{i}-\tau_{a c t}^{i}\right)^{2}}{M}}
$$

where $M$ is the total number of samples. Moreover, $\tau_{c a l}^{i}$ and $\tau_{a c t}^{i}$ are the calculated and actual values of the $i$ th point, respectively.

\section{Designing the excitation trajectory}

Studies show that a well-designed excitation trajectory can not only reduce the adverse impact of measurement noise on the result but also improves the iterative speed of the target parameter and the identification accuracy (Shouyan et al., 2018). Designing the excitation trajectory generally consists of two steps, including the trajectory parameter selection and the parameter optimization.

\subsection{Trajectory parametrization}

The trajectory of each joint can be considered as the finite sum of the Nth harmonic sine and cosine functions. The position curve of the $i$ th joint $q_{i}$, speed $\dot{q}_{i}$, and the acceleration $\ddot{q}_{i}$ can be expressed as follows.

$$
\begin{aligned}
& q_{i}(t)=\sum_{l=1}^{N} \frac{a_{l}}{w_{f} l} \sin \left(\omega_{f} l t\right)-\frac{b_{l}}{\omega_{f} l} \cos \left(\omega_{f} l t\right)+q_{i 0} \\
& \dot{q}_{i}(t)=\sum_{l=1}^{N} a_{l} \cos \left(\omega_{f} l t\right)+b_{l} \sin \left(\omega_{f} l t\right) \\
& \ddot{q}_{i}(t)=\omega_{f} \sum_{l=1}^{N} b_{l} l \cos \left(\omega_{f} l t\right)-a_{l} l \sin \left(\omega_{f} l t\right)
\end{aligned}
$$

where $\omega_{f}$ is the fundamental frequency, and in the present study, it is set to $0.1 \pi(\mathrm{rad} / \mathrm{s})$. Moreover, $q_{i 0}$ denotes the offset of the joint position. In order to ensure the periodicity of the trajectory, it is assumed that all joints have the same fundamental frequency. Each track contains $(2 \mathrm{~N}+1)$ parameters $(\mathrm{N}$ is the terms of Fourier series) to generate the excitation reference trajectory. The parameters $a_{l}$ and $b_{l}$ determine the magnitude of the cosine and sine functions and can be determined through the optimization.

\subsection{Trajectory optimization}

The problem of determining the excitation trajectory 
$q^{*}(t)$ can be mathematically expressed as follows:

$$
q^{*}(t)=\arg \min \left(O_{J}\right)
$$

Meanwhile, this expression is subjected to the following constraints and boundary conditions:

$$
\begin{cases}\left|q_{i}(t)\right| \leq q_{\max } & (a) \\ \left|\dot{q}_{i}(t)\right| \leq v_{\max } & (b) \\ \left|\ddot{q}_{i}(t)\right| \leq a_{\max } & (c) \\ q_{i}\left(t_{0}\right)=q_{i}\left(t_{f}\right)=0 & (d) \\ \dot{q}_{i}\left(t_{0}\right)=\dot{q}_{i}\left(t_{f}\right)=0 & (\mathrm{e}) \\ \ddot{q}_{i}\left(t_{0}\right)=\ddot{q}_{i}\left(t_{f}\right)=0 & (f)\end{cases}
$$

where $O_{J}$ is the objective function to be determined. $q_{\max }, v_{\max }$ and $a_{\max }$ are the position, velocity and acceleration of the maximum, respectively. Boundary conditions (e) and (f) limit the starting velocity and acceleration of the robot's excitation trajectory, respectively.

Based on Hadamard's inequality.

$$
\begin{aligned}
\operatorname{det}\left(W^{T} W\right) & \leq \sum_{i=1}^{n M} W_{i 1}^{2} \ldots \sum_{i=1}^{n M} W_{i N_{b}}^{2} \\
& \leq \prod_{j=1}^{N_{b}} \sum_{i=1}^{n M} W_{i j}^{2}
\end{aligned}
$$

Defining $\sum_{i=1}^{n M} W_{i j}^{2}=W_{j}^{s}$ then equation (28) can be simplified to the form below:

$$
\operatorname{det}\left(W^{T} W\right) \leq \prod_{j=1}^{N_{b}} W_{j}^{s}
$$

Then the objective function is defined as follows:

$$
O_{J}=\frac{1}{\prod_{j=1}^{N_{b}} \sum_{i=1}^{n M} W_{i j}^{2}}
$$

Where the matrix $\bar{W}_{1}$ in equation (11) will be used as $W$. Assuming $a_{l} \neq 0, \quad b_{l} \neq 0$ and $N=5,66$ excitation trajectory parameters of the 6-DOF robot can be obtained by the NLOPT optimization method. The period of excitation trajectory is 20 seconds. Figure 1 presents the excitation trajectories of 6 joints.

Figure 1 Position of the reference trajectory

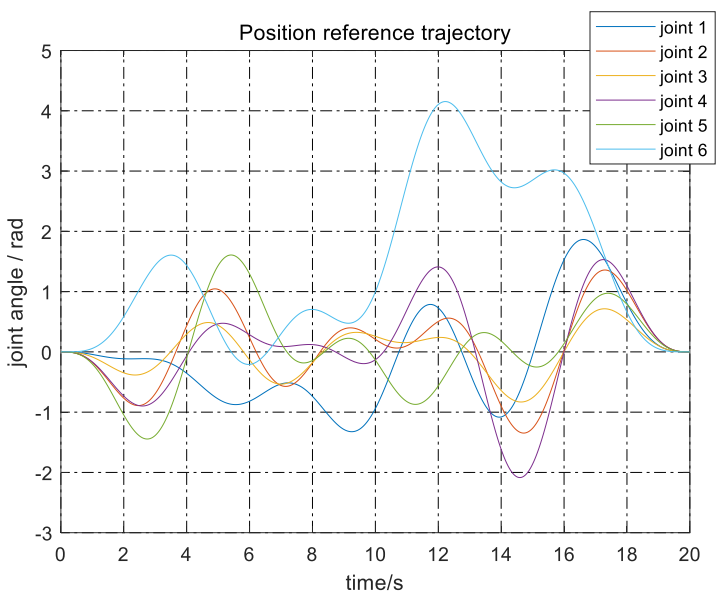

\section{Sensor-less hand guiding of the industrial robot}

Sensor-less hand guiding of the industrial robot is one of the typical applications, which requires accurate dynamic parameters (Fan et al., 2019). Recently, a sensor-less force control method has been proposed based on the motor current for the direct drive robot (Sang et at., 2016). Furthermore, an effective technique was applied for the sensor-less estimation of the force, which is applied to the end-effector of a robot using the stiffness and relative displacement of each robot joint (Yuan et al., 2018). However, these approaches are only applicable to special robots equipped with joint torque sensors or direct drive motors.

The core idea behind the sensor-less hand guiding is to accurately estimate the robot inertia, gravity and friction without joints' torque sensors so that the programmer can compute a feedforward controller to compensate such items while the robot is moving. Finally, the robot movement can be directly guided with a small external force. Based on the foregoing discussions, it is an enormous challenge to calculate the friction in all cases. This is especially more pronounced when the joint state changes from moving to stationary, or slow movement. The most accurately identified parameters are the smaller external force required for the hand guiding. Therefore, the hand guiding is carried out in the present study to evaluate the accuracy of the identified parameters in practical applications.

Based on the results of dynamic identification parameters, the real-time torque of each joint can be accurately calculated from the expression (31) (Sang et al., 2016). 


$$
\begin{aligned}
\tau= & {\left[n^{2} \hat{J}+\hat{M}(q)\right] \ddot{q}_{d}+\hat{C}(q, \dot{q}) \dot{q}+\hat{G}(q) } \\
& +\hat{\tau}_{f}+K_{d}\left(\dot{q}_{d}-\dot{q}\right)+K_{p}\left(q_{d}-q\right)
\end{aligned}
$$

where $\tau$ is the torque vector of joints, $K_{p}$ and $K_{d}$ are gain matrixes of PD controller. Moreover, $\hat{J}, \hat{M}(q)$, $\hat{C}(q, \dot{q}), \hat{G}(q)$ and $\hat{\tau}_{f}$ are the estimated values for motor inertia moment, inertia matrix, Coriolis and centrifugal matrix, vector of gravitational torque and joint friction vector, respectively. Meanwhile, $q_{d}, \dot{q}_{d}$ and $\ddot{q}_{d}$ are the desired position, velocity and acceleration of joints, respectively. It should be indicated that for the hand guiding, the proportional control term should be excluded from equation (31), otherwise the position control action will drive the robot to the desired position. Furthermore, the desired velocity and acceleration of each joint should be zero. For the sake of convenience, the influence of the inertia term is ignored in the present study. Figure 2 shows that since the inertia term is ignored, this strategy is suitable for low-speed and low-acceleration hand guiding, and the coefficient matrices or vectors can be calculated with robot dynamic parameters.

$$
\tau=\left\{C(q, \dot{q}) \dot{q}+G(q)+\tau_{f}\right\}-K_{d} \dot{q}
$$

Figure 2 Block diagram of the sensor-less hand guiding

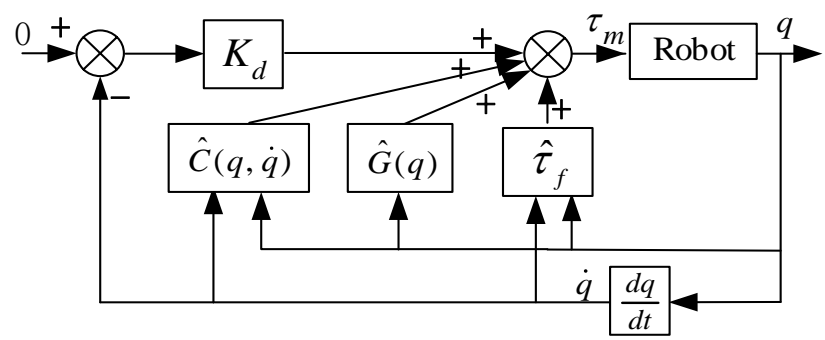

\section{Calculation and verification of dynamic parameters}

\subsection{Experiment Platform}

Figure 3 shows the robot experiment platform, which includes an industrial manipulator, a servo system to drive the motors, and a real-time main controller. In order to visualize the data, debug the system and modify the controller algorithm, an internally developed programming interface is used (Guanghui et al., 2019). Figure 4 shows the kinematic structure and parameters of the industrial manipulator. The weight and maximum payloads are approximately $47 \mathrm{~kg}$ and $7 \mathrm{~kg}$, respectively. Moreover, the communication protocol between the controller and the motor driver is EtherCAT, and the frequency is $1 \mathrm{kHz}$.
In the robot, there is not a torque senor in every joint. The estimation value is computed according to joint's current. The estimated torques are used for modelling the friction and estimating the dynamic parameters of the robot.

Figure 3 Experiment platform

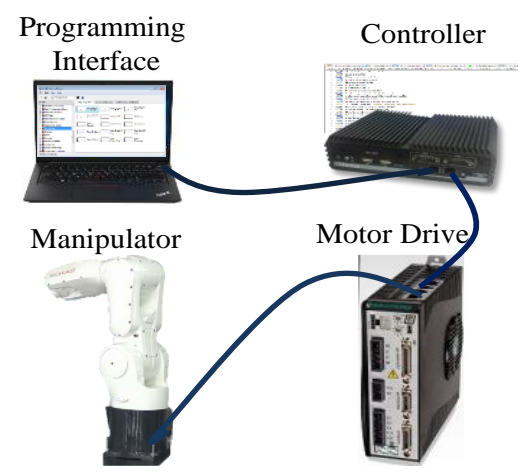

Figure 4 ROKAE BX7 robot mechanism diagram

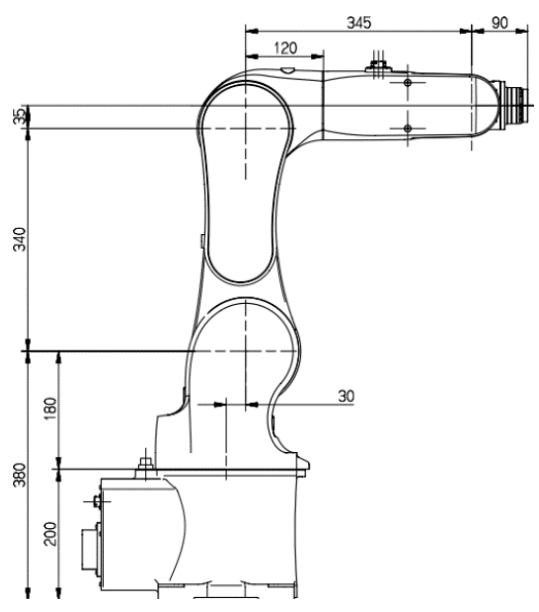

\subsection{Estimation of dynamic parameters}

According to section 2, the number of parameters that should be identified for each joint is 16 and the total number of parameters required for the 6-DOF robot is 96. Figure 5 illustrates the identification process.

Figure 5 Dynamic parameters identification diagram

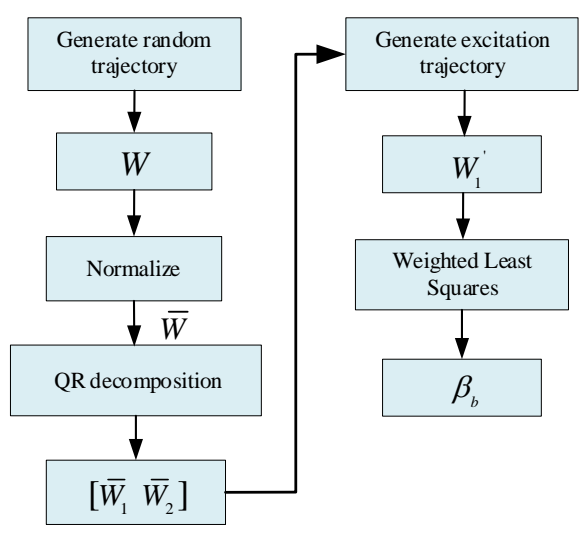


The whole pipeline of the identification is as the following. Firstly, joint position, velocity and acceleration are collected under the random trajectory (six sine curves are used in this paper) and the coefficient matrix $W$ is obtained. After the matrix $W$ is normalized and the $\mathrm{QR}$ is decomposed, $W$ can be divided into $\bar{W}_{1}$ and $\bar{W}_{2}$. The matrix $\bar{W}_{1}$ is used to generate the excitation trajectory.

Secondly, running the robot under the excitation trajectory and 20,000 data groups of position, velocity and acceleration of the joints are collected and twenty thousand matrixes $Y_{s}^{\prime} \in R^{6 \times 96}$ can be obtained like equation (8). Concatenating $Y_{s}$ matrixes, the observation matrix $W^{\prime} \in R^{120000 \times 96}$ is obtained. By extracting corresponding columns like equation (11), the matrix $W^{\prime}$ is divided into $W_{1}^{\prime} \in R^{120000 \times 70}$ and $W_{2}^{\prime} \in R^{120000 \times 26}$. Where, 70 and 26 are the number of linearly independent and dependent vectors, respectively.

Finally, $\beta_{b}$ is calculated by the weighted least squares in equation (23). Multiple solutions exist for the vectors $\beta_{s}^{\prime}$ and $\beta_{s}^{\prime \prime}$ in equation (18). One way to solve this linear system is to assign specific values to the elements of $\beta_{s}^{\prime \prime}$. In standard parameters set $\beta_{s}$, elements $m_{i} c_{m_{i} x}$, $m_{i} c_{m_{i} y}$ and $m_{i} c_{m_{i} z}$ contain $m_{i}$. Therefore, if the eigenvector corresponding to $m_{i}$ is not independent, $m_{i}$ cannot be set to 0 . Otherwise, the independency of $m_{i} c_{m_{i} x}, \quad m_{i} c_{m_{i} y}$, and $m_{i} c_{m_{i} z}$ cannot be judged. Then, in the mass item $m_{i}$ in $\beta_{s}^{\prime \prime}, m_{i}$ is set to 10 , and the rest elements are set to 0 . Using this method, a solution of equation (18) is obtained. Table I shows the identification parameters of the ROKAE BX7 robot.

Table I shows that all mass items of six joints are 10, which indicates that the eigenvectors corresponding to these mass items are not independent, and they are manually set to 10 . Moreover, the non-independent eigenvalues are set to 0 except for $m_{i}$ that is set to 10 .

It should be indicated that the parameters set obtained in this way is the numerical solution. It may not be completely consistent with the actual physical parameters of the joints. However, the experimental results show that this set of parameters can be used for computing model items correctly. Some intuitive hints can be used to qualitatively understand these parameters. For example, in joint 1 , the component of mass in three directions and the inertias are all 0 except in the $\mathrm{z}$ direction. This is because the joint 1 itself does not move except for rotation around the $\mathrm{z}$-axis during the movement.

Moreover, the parameters of the friction model are qualitatively evaluated. Table I shows that $f_{v 1}^{i}$ is higher than $f_{v 2}^{i}$ and $f_{v 3}^{i}$. This is reasonable because the conventional friction model only considers the velocity of the joint. Moreover $f_{v 2}^{i}$ and $f_{v 3}^{i}$ are not zero, which means that the joint friction is correlated to $\dot{q}^{2}$ and $\dot{q}^{3}$ as equation (4). The Coulomb friction parameters $f_{c 1}^{i}$ and $f_{c 2}^{i}$ in the positive and negative directions of each joint are approximately equal, which is in line with the actual situation. However, they are different, which confirms that it is correct to consider the Coulomb friction in positive and negative directions separately.

Table I The estimated dynamic parameters of ROKAE BX7

\begin{tabular}{ccccccc}
\hline Parameters & Joint 1 & Joint 2 & Joint 3 & Joint 4 & Joint 5 & Joint 6 \\
\hline$m_{i}(\mathrm{~kg})$ & 10 & 10 & 10 & 10 & 10 & 10 \\
$m_{i} c_{m_{i} x}(\mathrm{~kg} \cdot \mathrm{m})$ & 0.0000 & -858.2752 & -76.5142 & -0.2413 & 0.0616 & -1.5041 \\
$m_{i} c_{m_{i} y}(\mathrm{~kg} \cdot \mathrm{m})$ & 0.0000 & -5.2483 & -861.6599 & -3.3241 & 3.8733 & 1.5720 \\
$m_{i} c_{m_{i} z}(\mathrm{~kg} \cdot \mathrm{m})$ & 0.0000 & 0.0000 & 0.0000 & 0.0000 & 0.0000 & 0.0000 \\
$I_{x x}^{i}\left(k g \cdot \mathrm{m}^{2}\right)$ & 0.0000 & 3.9782 & -10.8293 & 0.0998 & 0.0595 & -0.0227 \\
$I_{x y}^{i}\left(\mathrm{~kg} \cdot \mathrm{m}^{2}\right)$ & 0.0000 & 0.3662 & 1.1390 & -0.0073 & -0.0048 & 0.0006 \\
$I_{x z}^{i}\left(\mathrm{~kg} \cdot \mathrm{m}^{2}\right)$ & 0.0000 & 0.5461 & -0.2281 & -0.0444 & -0.0125 & -0.0094 \\
$I_{y y}^{i}\left(\mathrm{~kg} \cdot \mathrm{m}^{2}\right)$ & 0.0000 & -7.3663 & -0.0585 & -0.0000 & -0.0000 & -0.0000 \\
$I_{y z}^{i}\left(\mathrm{~kg} \cdot \mathrm{m}^{2}\right)$ & 0.0000 & 0.1911 & -0.1043 & 0.0458 & -0.0011 & 0.0098 \\
$I_{z z}^{i}\left(k g \cdot \mathrm{m}^{2}\right)$ & -2.8121 & -9.3237 & -9.6584 & 0.0845 & 0.0884 & -0.012
\end{tabular}




\begin{tabular}{ccccccc}
$\boldsymbol{J}_{i}\left(\mathrm{~kg} \cdot \mathrm{m}^{2}\right)$ & 0.0000 & 0.0000 & -2.8085 & 0.1407 & -0.0429 & -0.0411 \\
$f_{v 1}^{i}(\mathrm{Nm} \cdot \mathrm{s} / \mathrm{rad})$ & 33.6469 & 35.4598 & 20.6053 & 6.7420 & 4.2719 & 0.7638 \\
$f_{v 2}^{i}(\mathrm{Nm} \cdot \mathrm{s} / \mathrm{rad})$ & 1.6392 & 1.2254 & 2.5514 & -0.0174 & 0.2154 & 0.0326 \\
$f_{v 3}^{i}(\mathrm{Nm} \cdot \mathrm{s} / \mathrm{rad})$ & -4.5485 & -5.5002 & -10.4831 & -0.3451 & -0.5393 & -0.0814 \\
$f_{c 1}^{i}(\mathrm{Nm} \cdot \mathrm{s} / \mathrm{rad})$ & 8.0499 & 13.8660 & 9.2687 & 3.9203 & 3.0459 & 0.9669 \\
$f_{c 2}^{i}(\mathrm{Nm} \cdot \mathrm{s} / \mathrm{rad})$ & -10.9926 & -13.3944 & -8.8085 & -3.8707 & -3.2091 & -0.9670 \\
\hline
\end{tabular}

\subsection{Validation and error analysis}

In this section, the proposed method is evaluated by comparing it with two different friction models and identification approaches as the following:

- The conventional friction model and least squares estimation method (TFLS).

- The modified friction model and least squares estimation method (MFLS).
- The modified friction model weighted least squares estimation method (MFWLS) proposed in this study.

All of the approaches are tested on the same platform with the same test trajectory. Moreover, the actual torques of joints are compared with calculated torques. Figures 6,7and 8 show the obtained results. Table II lists the root mean square error (RMSE) values of these methods.

Figure 6 Dynamic model validation curves based on the TFLS
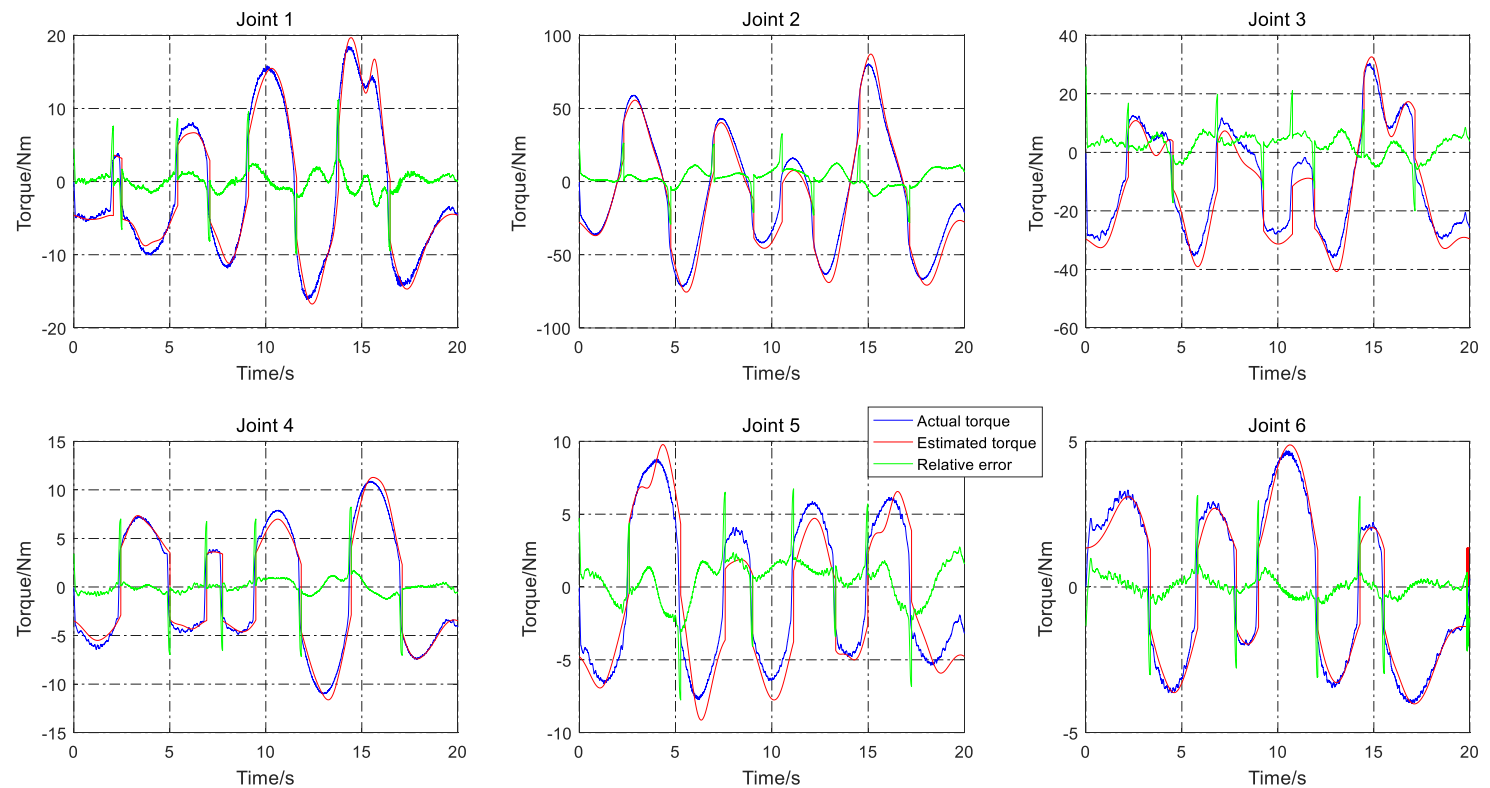

Figure 7 Dynamic model validation curves based on MFLS 

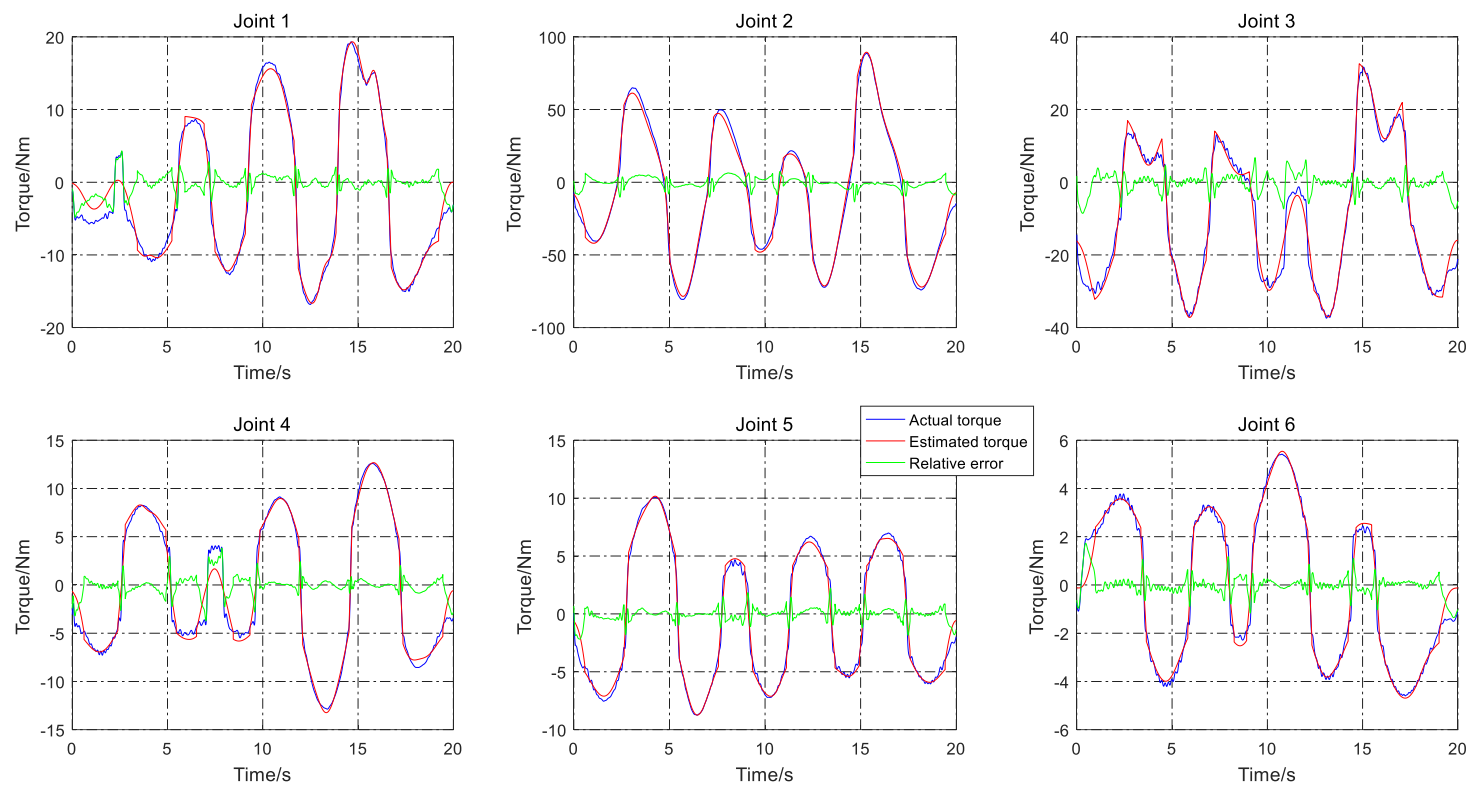

Figure 8 Dynamic model validation curves based on the MFWLS
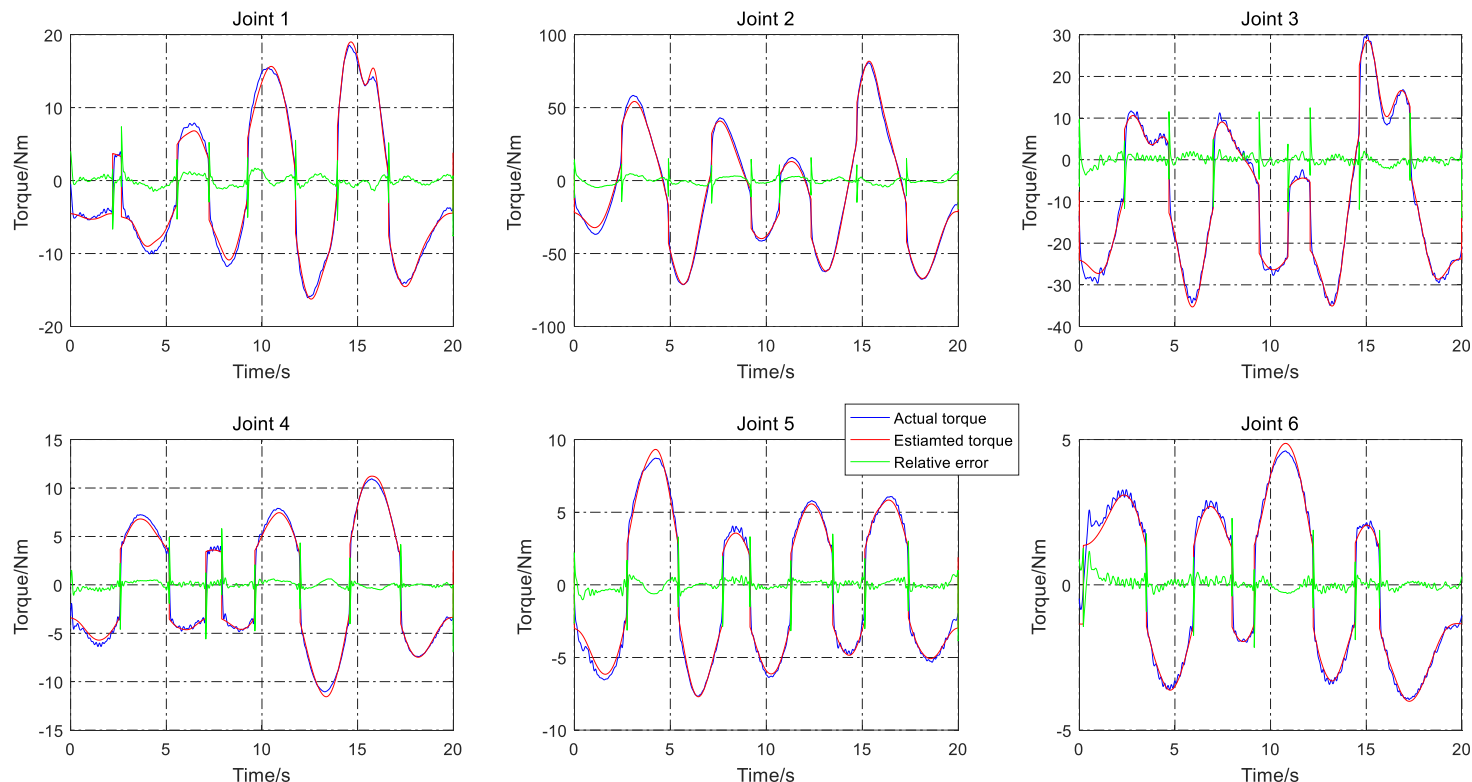

Table II Root Mean Square Error of three methods

\begin{tabular}{cccc}
\hline Joint & TFLS & MFLS & MFWLS \\
\hline 1 & 1.1591 & 1.1229 & 0.4874 \\
2 & 16.1487 & 15.5289 & 14.9412 \\
3 & 23.6079 & 13.9014 & 4.0292 \\
4 & 1.5134 & 1.6229 & 0.2823 \\
5 & 1.0070 & 0.9462 & 0.3320 \\
6 & 0.9255 & 0.7810 & 0.2702 \\
\hline
\end{tabular}

Figures 6 and 7 show the comparison between the estimated and actual values obtained by employing the TFLS and the MFLS, respectively. In comparison to the
TFLS, estimated errors of joints torque by the MFLS is effectively reduced, especially when the joints are switching directions, which demonstrates that the modified friction describes the robot joint friction more accurately. This is consistent with the results in Table II. Moreover, the RMSE of the MFLS method is lower than that of the TFLS method from joint 1 to 3. Furthermore, a modified friction model effectively reduces "jumping" of the estimated values when the torque is close to zero. However, it is observed that the RMSE of the last three joints is not significantly reduced comparing compared with the first three joints. This is mainly because the least square estimation method does not consider the difference in the output torque of different joints as 
analyzed in section 2.3. Figure 8 and Table II show that the estimated accuracies of all joints are improved by the MFWLS method proposed in this paper compared with the TFLS method. Moreover, the average of six joints RMSE decreases more than 54\%, which proves the validity of the MFWLS method proposed in this study.

\subsection{Hand guiding experiment and analysis}

In this section, robot hand guiding is used to verify the practicability of the identified parameters in this study, and to confirm the advantage of the modified friction model over the conventional friction model in practice. Figure 9 shows that there are some marks on the carton, and the experimenter drags the robot with one hand to move to each marked point. The torque command of the robot consists of a full torque compensation calculated by the part in curly braces of equation (32) and the output of the velocity controller.

Figure 9 Robot hand guiding experiment
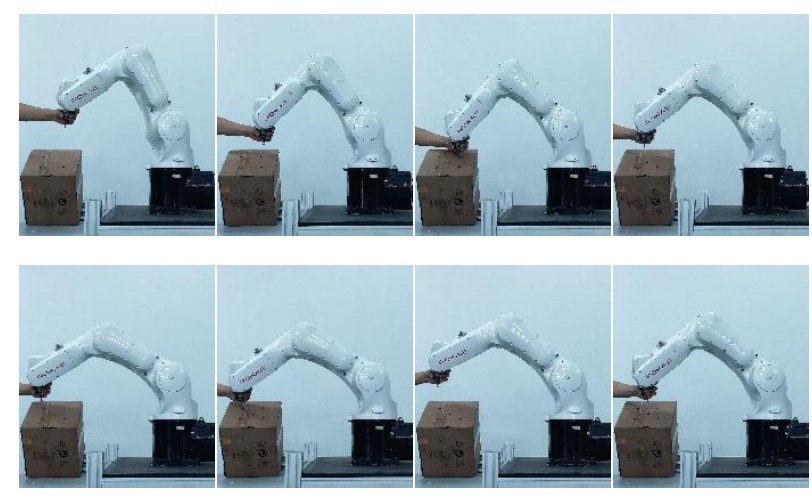

Figure 10 shows the hand guiding the analysis of the industrial robot. In Figure 10 (a), the "Compensation torque / TFLS" curve is the full compensation torque calculated by the part in Curly braces of equation (32) with the TFLS method in the process of dragging robot, and while the "Compensation torque / MFWLS" curve is based on the MFWLS method, correspondingly. The last curve is output torque of motor 3 . It should be indicated that only the result with the data of joint 3 is demonstrated due to the limited space. Obtained results from the other joints are similar. It is observed that at the initial moment, the robot is stationary, and the output torque of the motor is negative to overcome the gravity of the arm. Moreover, the compensation torque calculated based on the MFWLS method is basically the same as the motor output, while the compensation torque calculated based on the TFLS method is higher. From 2.5 seconds onwards, an external force is applied to the robot, and the robot starts to move. According to the principle of the force-balance, the external torque of the joint 3 can be calculated by subtracting the output torque of the motor from the compensation torque. In Figure 10 (b), the "External torque / TFLS" curve and the "External torque / MFWLS" curve show the required external torque to the joint when dragging the robot using the TFLS method and the MFWLS method, respectively. Moreover, the "Velocity of joint 3" curve is the velocity of the joint 3 in the process of dragging, whose units are shown on the right scale of figure 10(b). According to the parameters identified by the TFLS method, the required external torque at low joint speed ( $\mid$ velocity $\mid \leq 0.01 \mathrm{rad} / \mathrm{s})$ is higher than the result of MFWLS method, and the external torque curve is not smooth enough. In comparison, the MFWLS-based method requires less external torque for the robot, whether it is stationary, at low or high speed (|velocity $\mid>$ $0.01 \mathrm{rad} / \mathrm{s}$ ). More specifically, the external torque required by the robot from being motionless to having motion will be smoother, which verifies the practicability of the parameters identified using the MFWLS method and the advantage of the modified friction model over the previous model.

Figure 10 Hand guiding analysis of the industrial robot
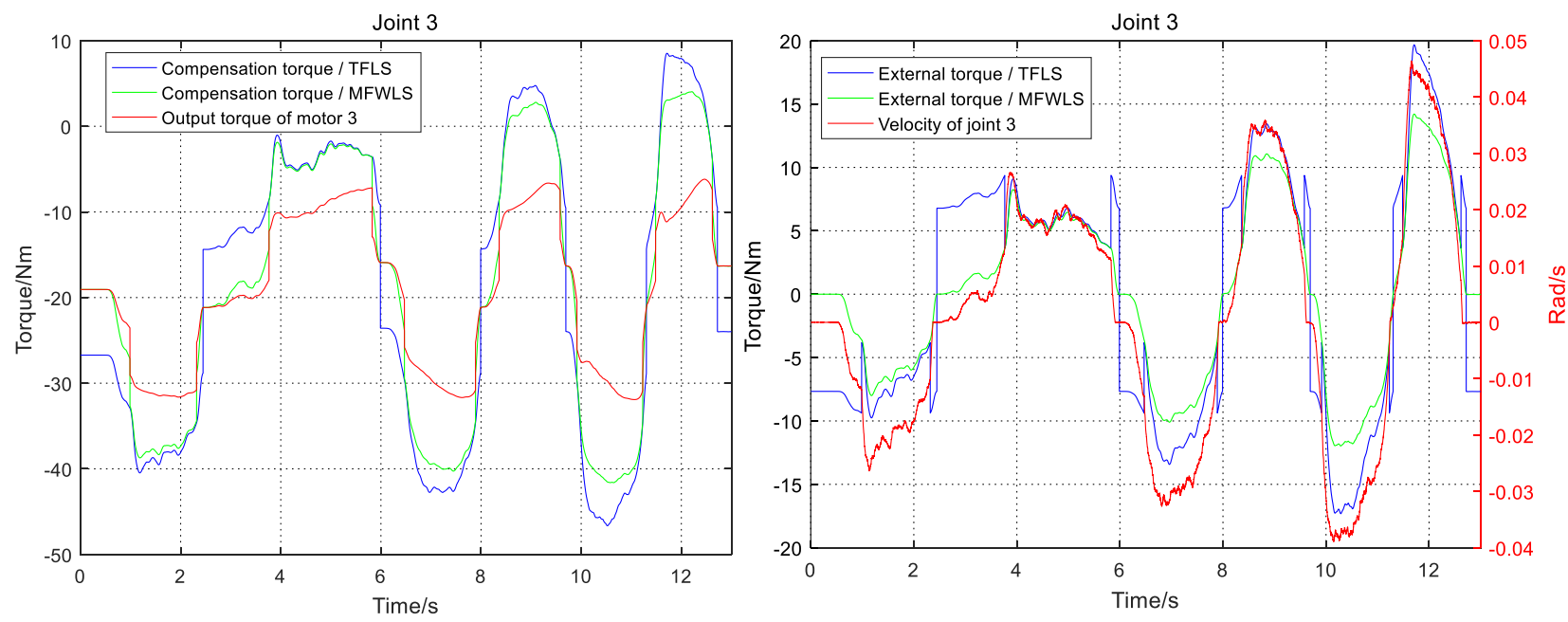


\section{Conclusion}

In the present study, a modified friction model is proposed to accurately describe the robot friction model. Moreover, a weighted least squares scheme and an efficient solution to the dynamic equation are introduced. Experimental results demonstrate that the abovementioned improvements effectively improve the accuracy of dynamic parameter estimation. Furthermore, a hand guiding experiment is carried out using the estimated dynamic parameters. It provides an applicable sensor-less hand guiding scheme for the industrial robot and validates the availability of the dynamic parameter identification method in this study. In the future, we will attempt to establish an accurate friction model to describe the conversion of dynamic and static friction. In addition, we will study the interactive control based on the identified dynamic parameters.

\section{References}

Afrough, M., Hanieh, A.A. (2018), "Identification of dynamic parameters and friction coefficients", Journal of Intelligent \& Robotic Systems, Vol. 2, pp. 1-11.

Bittencourt, A.C., Wernholt, E. and Sander-Tavallaey, S. (2010), "An extended friction model to capture load and temperature effects in robot joints", IEEE/RSJ International Conference on Intelligent Robots and Systems, pp. 6161-6167.

Bona, B., Indri, M. (2005), "Friction compensation in robotics: an overview", In Proceedings of the 44th IEEE Conference on Decision and Control, pp. 4360-4367.

Craig, J.J. (2005), "Introduction to Robotics: Mechanics and Control, Addison-Wesley", Boston, MA, pp. 263-264.

Fan, Z., Juliang, X. and Haitao, L. (2019), "Force/Torque Sensorless Compliant Control Strategy for Assembly Tasks Using a 6-DOF Collaborative Robot”, IEEE ACESS, Vol. 7, 2019, pp.108795-108805.

Yuan, J., Wang, S.and Liang., Y. (2018), "Direct teaching of industrial manipulators using current sensors", Assembly Automation, Vol. 38 No. 2, pp. 216-225.

Gautier, M., Janot, A. and Vandanjon, P, O. (2013). “A new closed loop output error method for parameter identification of robot dynamics", IEEE Trans Control Syst Technol, 21:428-44.

Guanghui, L., Bing, H. and Qingxin, L. (2019), "DOREP: An Educational Experiment Platform for Robot Control Based on MATLAB”, ICIRA 2019, LNAI 11745, pp.555-565.

Jing, B., Le, F. and Shuyang, Z. (2019), "The parameter identification model considering both geometric parameters and joint stiffness", Industrial Robot: the international journal of robotics and application 47/176-81.

Jingfu, J., Nicholas, G. (2015), "Parameter Identification for Industrial Robots with a Fast and Robust Trajectory Design Approach", Robotics and Computer-Integrated Manufacturing, 31, 2015, pp. 21-29.

Juliang, X., Fan, Z. and Qiulong, Z. (2019), "Research on the forcefree control of cooperative robots based on dynamic parameters identification", Industrial Robot: the international journal of robotics and application 46/4 499-509.

Juliang, X., Qiulong, Z. and Ying, H. (2018), “Collision detection algorithm for collaborative robots considering joint friction",International Journal of Advanced Robotic Systems, Vol. 15, No.4 (2018) ISSN 1729-8806, pp.1-13.

Kubus, D., Krger, T. and Wahl, F. (2008), "On-line estimation of inertial parameters using a recursive total least-squares approach", Intelligent Robots and Systems,IEEE/RSJ International Conference on, 2008, pp. 3845-52.

Matthias, N., Hubert, G. and Hartmut, B. (2015), "A persistent method for parameter identification of a seven-axes manipulator", Robotica, volume 33, pp. 1099-1112.

Nevmerzhitskiy, M. N., Notkin, B. S. and Vara, A. V. (2019), "Friction Model of Industrial Robot Joint with Temperature Correction by Example of KUKA KR10", Journal of Robotics, 2019, pp.1-11.

Olsson, H., Åström, K. J. and De Wit, C.C. (1998), "Friction models and friction compensation", European Journal of Control, 4(3): 176-195.

Sami, H., Alessandro D.L., and Albu, S. (2017). "Robot Collisions: A Survey on Detection, Isolation, and Identification," IEEE TRANSACTIONS ON ROBOTICS, pp.121.

Sang-Duck, L., Kulk-Hyun, A. and Jae-Bok, S. (2016), “Torque Control based Sensor-less Hand Guiding for Direct Robot Teaching”, 2016 IEEE/RSJ International Conference on Intelligent Robots and Systems (IROS), pp.745-750.

Shouyan, C., Tie, Z. (2018), "Force control approaches research for robotic machining based on particle swarm optimization and adaptive iteration algorithms", Industrial Robot, Vol. 45 No. 1, pp.141-151.

Swevers, J., Ganseman, C., Tukel, D. and Joris, S. (1997), "Optimal robot excitation and identification", IEEE Trans Robot Autom, 13:730-40.

Swevers, J., Verdonck, Walter. and Ang, Schutter, J.D. (2007), "Dynamic model identification for industrial robots", IEEE Control Systems, 27(5), 58-71.

Waiboer, R., Aarts, R. and Jonker, B. (2005), "Velocity dependence of joint friction in robotic manipulators with gear transmissions", In ECCOMAS Thematic Conference Multibody Dynamics.

Wu, J., Wang, J. and You, Z. (2010), “An overview of dynamic parameter identification of robots", Robotics and ComputerIntegrated Manufacturing, Vol. 26 No. 5, pp.414-419. 